

\title{
A Transverse Spectrum Deconvolution Technique for MIMO Short- Range Fourier Imaging
}

Fromenteze, T., Yurduseven, O., Berland, F., Decroze, C., Smith, D., \& Yarovoy, A. (2019). A Transverse Spectrum Deconvolution Technique for MIMO Short-Range Fourier Imaging. IEEE Transactions on Geoscience and Remote Sensing. https://doi.org/10.1109/TGRS.2019.2905269

Published in:

IEEE Transactions on Geoscience and Remote Sensing

Document Version:

Peer reviewed version

Queen's University Belfast - Research Portal:

Link to publication record in Queen's University Belfast Research Portal

Publisher rights

(c) 2019 IEEE.

This work is made available online in accordance with the publisher's policies. Please refer to any applicable terms of use of the publisher.

\section{General rights}

Copyright for the publications made accessible via the Queen's University Belfast Research Portal is retained by the author(s) and / or other copyright owners and it is a condition of accessing these publications that users recognise and abide by the legal requirements associated with these rights.

Take down policy

The Research Portal is Queen's institutional repository that provides access to Queen's research output. Every effort has been made to ensure that content in the Research Portal does not infringe any person's rights, or applicable UK laws. If you discover content in the Research Portal that you believe breaches copyright or violates any law, please contact openaccess@qub.ac.uk. 


\title{
A Transverse Spectrum Deconvolution Technique for MIMO Short-Range Fourier Imaging
}

\author{
Thomas Fromenteze $^{\circledR}$, Okan Yurduseven ${ }^{\circledR}$, Senior Member, IEEE, Fabien Berland, Cyril Decroze, \\ David R. Smith, Member, IEEE, and Alexander G. Yarovoy, Fellow, IEEE
}

\begin{abstract}
The growing need for high-performance imaging tools for terrorist threat detection and medical diagnosis has led to the development of new active architectures in the microwave and millimeter range. Notably, multiple-input multiple-output systems can meet the resolution constraints imposed by these applications by creating large, synthetic radiating apertures with a limited number of antennas used independently in transmitting and receiving signals. However, the implementation of such systems is coupled with strong constraints in the software layer, requiring the development of reconstruction techniques capable of interrogating the observed scene by optimizing both the resolution of images reconstructed in two or three dimensions and the associated computation times. In this paper, we first review the formalisms and constraints associated with each application by taking stock of efficient processing techniques based on spectral decompositions, and then, we present a new technique called the transverse spectrum deconvolution range migration algorithm allowing us to carry out reconstructions that are both faster and more accurate than with conventional Fourier domain processing techniques. This paper is particularly relevant to the development of new computational imaging tools that require, even more pronouncedly than in the case of conventional architectures, fast image computing techniques despite a very large number of radiating elements interrogating the scene to be imaged.
\end{abstract}

Index Terms-MIMO radar, microwave imaging, millimeter wave radar, multistatic radar.

\section{INTRODUCTION}

I N MANY civilian and military applications, regions of interest need to be surveyed to gather useful information that is invisible to optical imaging systems. The use of electromagnetic radiation is often justified in these practical scenarios where nondestructive detection is required, measuring the interaction of the waves with the medium under

Manuscript received May 24, 2018; revised January 29, 2019; accepted March 7, 2019. This work was supported by the French National Research Agency (ANR PIXEL and ANR OBIWAM). (Corresponding author: Thomas Fromenteze.)

T. Fromenteze, F. Berland, and C. Decroze are with the University of Limoges, XLIM, UMR 7252, F-87000 Limoges, France (e-mail: thomas.fromenteze@unilim.fr).

O. Yurduseven is with the Centre for Wireless Innovation (CWI), Institute of Electronics, Communications and Information Technology (ECIT), School of Electronics, Electrical Engineering and Computer Science (EEECS), Queen's University Belfast, Belfast BT3 9DT, U.K.

D. R. Smith is with the Center for Metamaterials and Integrated Plasmonics, Duke University, Durham, NC 27708 USA.

A. Yarovoy is with the Microwave Sensing, Signals and Systems, Delft University of Technology, 2628 CD Delft, The Netherlands.

Color versions of one or more of the figures in this paper are available online at http://ieeexplore.ieee.org.

Digital Object Identifier 10.1109/TGRS.2019.2905269 test. In particular, the microwave range is of interest for imaging and has many advantages over other frequency bands, benefiting from the advanced maturity of the active devices required for the generation and measurement of waves and the high transmittance coefficient of common materials compared to that of the optical domain. The resolution of imaging systems is related to the diversity of information measured in time and space. Ideally, broadband and densely sampled antenna arrays emitting high-frequency and ultrawideband signals should, therefore, be used to minimize the size of point spread functions in range and cross-range [1]. However, the hardware complexity inherent in signal generation and measurement is dissuasive in many applications. This problem is exacerbated by multiple-input multiple-output (MIMO) systems, where independent transmitters and receivers are needed to improve the resolution of radar images compared with single-input multiple-outputs (SIMO) and multiple-input single-output (MISO) systems of the same number of radiating elements [2]. In this paper, innovative techniques have been proposed in recent years to take advantage of these constraints and increase the efficiency of imaging systems. A first category of solutions is based on the recent introduction of compressive sensing allowing for the efficient implementation of sparse arrays [3]-[5]. This approach exploits the inherent spatial structure of the imaged objects and scenes to reduce the number of signal samples measured over time, frequency, and space. A second category is based on the use of frequency-diverse radiating structures capable of encoding and multiplexing transmitted and/or received signals into a reduced number of compressed waveforms [6]-[10]. Simplifying the hardware associated with imaging systems, MIMO architectures can be more realistically applied to many applications requiring a large number of radiating elements interacting with each other, providing both fast measurements and access to high-resolution images.

Recent advances in MIMO imaging by the scientific community-particularly in the constrained framework of short-range applications-have made it possible to significantly improve the speed of associated imaging algorithms. These techniques, initially applied to SAR, SIMO, and MISO systems, are notably based on the spectral decomposition of measured radiation, taking advantage of the use of fast Fourier transforms implemented in this paper. Given the dispersion relation of plane waves deduced from the wave equation, rapid backpropagation can therefore be calculated in $k$-space by a technique called the range migration 


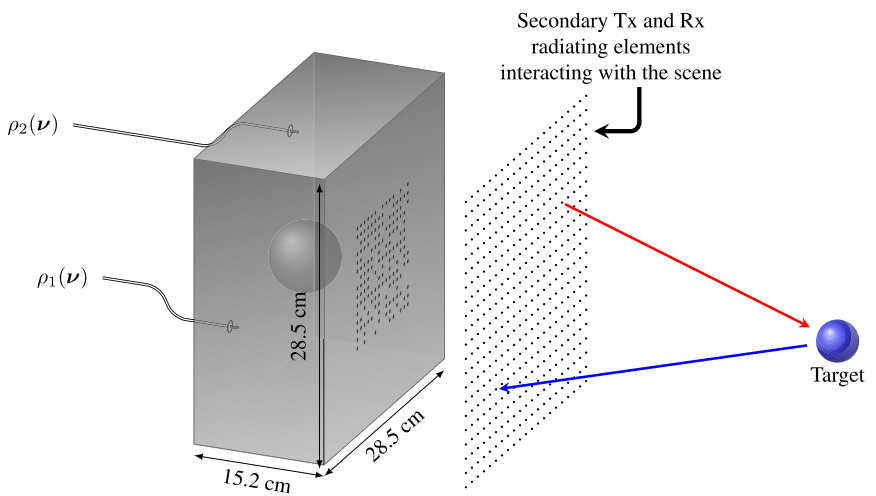

Fig. 1. Illustration of a computational imaging system operating in the microwave range [16]-[18]. An advanced characterization of the near field radiated by this metasurface opens access to the interaction with a set of secondary sources placed in the scanned aperture. This decomposition allows the implementation of rapid reconstruction techniques initially developed for conventional MIMO architectures.

algorithm (RMA) [11], [12]. This approach is particularly effective when combined with Stolt interpolations to rapidly transform the frequency dimension of the measured signals into range information, however introducing sometimes distortions in the computed images. In [13], this principle is extended to short-range MIMO systems, deriving an appropriate dispersion relation from a stationary phase approximation of the measured signal expression. This analysis will serve as a central pillar for the method proposed in this paper, showing that a physical interpretation of the interaction between the plane waves emitted and received by the MIMO array with the Fourier domain reflectivity function of the imaged scene can allow faster reconstructions, less memory-intensive, and of comparable quality. It is also interesting to be able to propose more intuitive tools in order to facilitate the comprehensionand possibly the teaching - of the complex processing techniques applied in this paper. An illustration based on the use of Moiré patterns is thus proposed in this framework to represent the asymptotic developments of complex integrals on which this technique is based.

The motivations for this paper are also based on the emerging field of computational imaging, where problems similar to those encountered in the case of conventional MIMO architectures are studied. Frequency-diverse radiating metasurfaces are engineered in this paper to achieve the coding and multiplexing of the transmit and receive waves into a limited number of frequency-dependent signals (Fig. 1). It has been shown in [14] and [15] that it is possible to factorize the sensing matrix encountered in computational imaging applications to separate the reconstruction stage of compressed signals from the backpropagation stage. This trick allows the implementation of conventional techniques whose formidable efficiency is based on the implementation of fast Fourier transforms. Although the full development of mathematical derivations associated with these computational techniques is outside the scope of this paper, it seemed important to justify the importance of developing new advanced processing techniques.

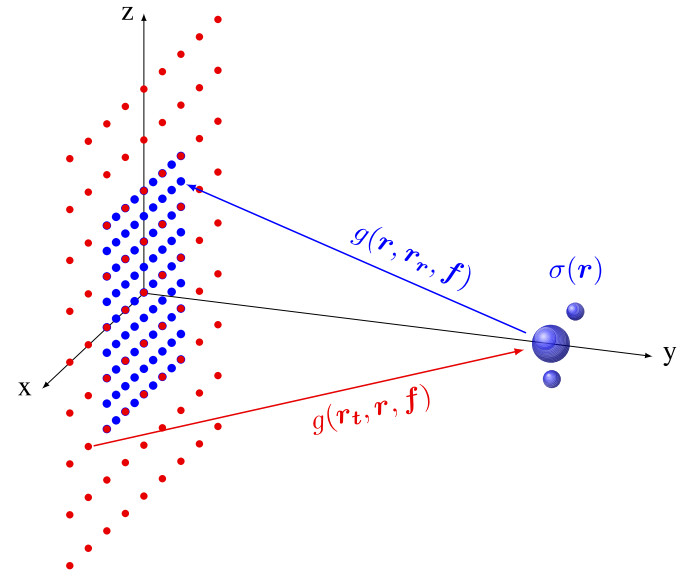

Fig. 2. Illustration of an MIMO array imaging setup. The interactions between the transmitting and receiving elements are measured to retrieve the target reflectivity function $\sigma(r)$. The propagation between the two locations $r_{i}$ and $r_{j}$ is modeled using free-space 3-D Green's functions $g\left(r_{i}, r_{j}, f\right)$.

The rest of this paper is organized as follows. Section II is an introduction to the general formalism associated with MIMO imaging systems. Here, we confine ourselves to short-range applications, recalling in particular the principle and limitations of the RMA as described in [13]. A new technique is introduced in Section II. Its main objective is to overcome the limitations associated with the interpolation step necessary for the proper functioning of the RMA in its current form, proposing a new approach essentially based on a physical analysis of the way in which an MIMO system interrogates the spectrum of the object to be imaged in the region of interest. This technique is finally applied to various numerical simulations in order to highlight the contributions allowed by them and its possible limitations. Finally, Section IV will then provide an overview of all the elements introduced in this paper and identify elements that could lead to future studies to further improve MIMO imaging techniques.

\section{Mimo Near-Field Imaging Problem}

A generic MIMO setup is presented in this section for the introduction of the proposed image reconstruction method. Since most applications require a compatibility with ultrawideband signals to optimize the range resolution, this constraint will be considered for the validation of this algorithm. A couple of 2-D arrays are considered for this study (Fig. 2). The transmit array is made of $n_{x_{t}} \times n_{z_{t}}$ isotropic radiating elements uniformly spaced in the plane $y=0$ at locations defined by the vectors $x_{t}, z_{t}$. Reciprocally, the receive array is made of $n_{x_{r}} \times n_{z_{r}}$ uniformly spaced in the plane $y=0$ at locations defined by $x_{r}, z_{r}$. These antennas are interacting with a target centered at the location $\left(x_{c}, y_{c}, z_{c}\right)$.

The target reflectivity function $\sigma(r)$ can be estimated from the measured signals $s\left(x_{t}, z_{t}, x_{r}, z_{r}, f\right)$, accounting for the interaction between all possible pairs of transmitting and receiving elements at each frequency. Using the first-order Born approximation and a scalar approximation of Maxwell's equations, the expression of the measured signals can be 
expressed as

$$
s\left(x_{t}, z_{t}, x_{r}, z_{r}, f\right)=\int_{r} \frac{1}{16 \pi^{2}} \frac{e^{-j k R_{t}}}{R_{t}} \sigma(r) \frac{e^{-j k R_{r}}}{R_{r}} d^{3} r
$$

where $r$ is the vectorized target space defined by the triplet of coordinate $(x, y, z) . R_{t}$ and $R_{r}$ are the Euclidean distances between the antennas and the target space, given by

$$
\begin{aligned}
& R_{t}=\sqrt{\left(x_{t}-x\right)^{2}+y^{2}+\left(z_{t}-z\right)^{2}} \\
& R_{r}=\sqrt{\left(x_{r}-x\right)^{2}+y^{2}+\left(z_{r}-z\right)^{2}} .
\end{aligned}
$$

A first estimation of the reflectivity function can intuitively be obtained by implementing a Kirchoff migration. A double summation is computed over the radiating aperture spaces $r_{t}=$ $\left(x_{t}, z_{t}\right)$ and $r_{r}=\left(x_{r}, z_{r}\right)$ for each voxel $r$ of the scene for compensating the phase induced by the propagation

$$
\hat{\sigma}(r)=\iiint s\left(x_{t}, z_{t}, x_{r}, z_{r}, f\right) e^{j \frac{2 \pi f}{c}\left(R_{t}+R_{r}\right)} d r_{t} d r_{r} d f .
$$

Despite the apparent simplicity of implementing this method, the computational time required to obtain a 3-D image using large antenna arrays is prohibitive in many applications. It is particularly interesting in this paper to move toward the use of backpropagation techniques in the Fourier domain. It is then possible to formalize the interaction of the reflectivity function of the scene with the emitted and received plane waves. It is necessary to start by expressing the signals received in the plane wave domain by means of Fourier transforms

$$
S\left(k_{x_{t}}, k_{z_{t}}, k_{x_{r}}, k_{z_{r}}, k\right)=\mathfrak{F}_{4 D}\left(s\left(x_{t}, z_{t}, x_{r}, z_{r}, f\right)\right)
$$

where $\mathfrak{F}$ is the Fourier transform operator. The frequency dimension is expressed as a function of the wavenumber $k=$ $(2 \pi f / c)$. The stationary phase method used in [13], partially adapted from [12], leads to a simplified representation of the measured signal $S$, approximated in the $k$-space domain as

$$
\begin{aligned}
S=\frac{-\pi}{k_{y_{t}} k_{y_{r}}} \int_{r} \sigma(x, y, z) e^{-j\left(k_{x_{t}}+k_{x_{r}}\right) x} & \\
& e^{-j\left(k_{z t}+k_{z r}\right) z} e^{-j k_{y} y} d^{3} r
\end{aligned}
$$

with

$$
\begin{aligned}
k_{y_{t}} & =\sqrt{k^{2}-k_{x_{t}}^{2}-k_{z_{t}}^{2}} \\
k_{y_{r}} & =\sqrt{k^{2}-k_{x_{r}}^{2}-k_{z_{r}}^{2}} \\
k_{x} & =k_{x_{t}}+k_{x_{r}} \\
k_{y} & =k_{y_{t}}+k_{y_{r}} \\
k_{z} & =k_{z_{t}}+k_{z_{r}} .
\end{aligned}
$$

The reflectivity function $\hat{\sigma}_{\text {conv }}(x, y, z)$ can thus be estimated in this paper by computing the following 3-D Fourier transform:

$$
\begin{array}{r}
\hat{\sigma}_{\text {conv }}(x, y, z) \propto k_{y_{t}} k_{y_{r}} \int_{k_{x}} \int_{k_{y}} \int_{k_{z}} S\left(k_{x_{t}}, k_{z_{t}}, k_{x_{r}}, k_{z_{r}}, k\right) \\
e^{j k_{y} y} e^{j k_{x} x} e^{j k_{z} z} d k_{x} d k_{y} d k_{z}
\end{array}
$$

A 3-D representation of the target space can be obtained in the $k$-space by interpolating the 5-D matrix computed with a 4-D Fourier transform applied to the measured signals. This step allows for a merging of the transmitted and received plane

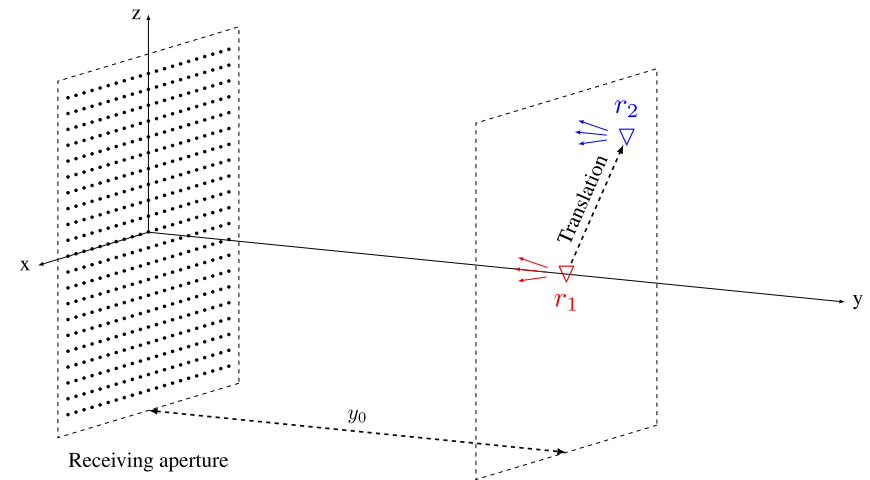

Fig. 3. Isotropic antenna is placed at two locations $r_{1}=\left(0, y_{0}, 0\right)$ and $r_{2}=\left(x_{2}, y_{0}, z_{2}\right)$, and signals $s(x, z) r_{i}$ are measured at the frequency $f_{0}$ by the radiating aperture for each position of the transmitter.

waves, considering the dispersion relation deduced from the stationary phase approximation developed in [13]. However, this last step is crucial and represents the most time-consuming process in this algorithm, especially for large arrays of unequal sizes where prior zero padding and spatial interpolation are required to work with suitable plane wave grids. Sparse arrays have been implemented in the MIMO systems to mitigate this computational limitation, exploiting the complementary spatial diversities of the transmit and receive arrays to ensure a full $k$-space coverage [19], [20] enabling the development of appropriate rapid processing techniques in this paper [21]. Furthermore, the recent development of compressive systems allowing for the multiplexing of a very large number of transmitted and received waveforms [14], [22] helped overcoming the hardware limitations inherent to high-resolution systems, leading to a growing need of efficient MIMO algorithms compatible with large and densely populated antenna arrays.

Before presenting a new technique to optimize the performance of the conventional MIMO RMA algorithm, it would seem useful to illustrate the origin of the efficiency of this technique, whose mathematical complexity tends to distract from an intuitive understanding. In this paper, a simple illustrative experiment is presented; a transmit antenna is placed facing a receive aperture (Fig. 3).

The measured signals are represented in the plane wave space to represent the impact of a source translation in a plane parallel to the aperture. In order to illustrate simply how the RMA works, this simplistic case, with no real practical value, is studied by calculating the spectrum of plane waves $S\left(k_{x}, k_{z}\right)_{r_{i}}=\mathfrak{F}_{2-D}\left(s(x, z)_{r_{i}}\right)$ of the signals received by the radiating aperture, for two locations $r_{1}=\left(0, y_{0}, 0\right)$ and $r_{2}=\left(x_{2}, y_{0}, z_{2}\right)$ of the isotropic source, for a given frequency $f_{0}$ corresponding to a wavenumber $k_{0}=2 \pi f_{0} / c$ (Fig. 4). The phase distribution computed for the two locations of the transmitter can be compared to that of range matching term $\exp \left(-j k_{y} y_{0}\right)$, where $k_{y}=\left(k_{0}^{2}-k_{x}^{2}-k_{z}^{2}\right)^{(1 / 2)}$ is the dispersion relation corresponding to this simple setup.

In this scenario, an RMA image reconstruction is performed by calculating the correlation between the resulting plane wave spectrum and a reference signal $\exp \left(-j k_{y} y_{0}\right)$ based on the dispersion relation, recalling the principle of holography [23]. 

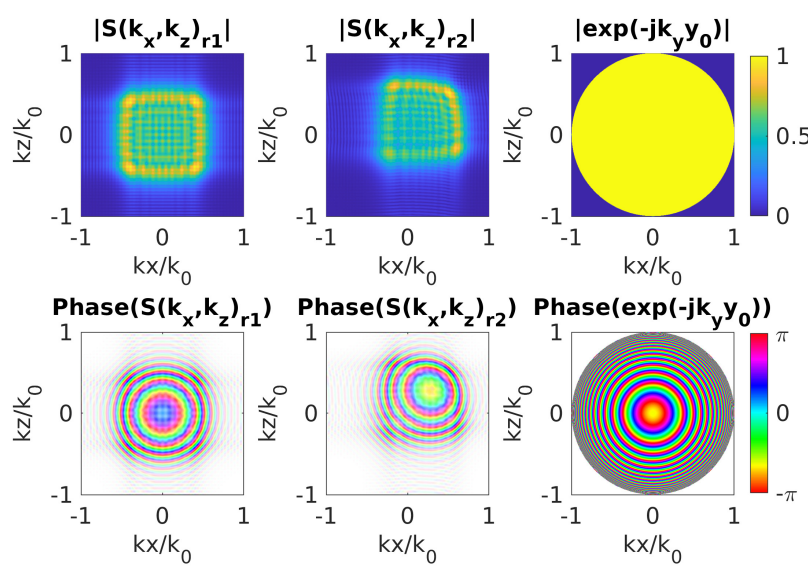

Fig. 4. Plane wave spectrum of the field measured by the radiating aperture for two positions $r_{1}$ and $r_{2}$ of transmitting antenna at a given frequency $f_{0}$ The RMA consists in calculating the correlation of this distribution with the reference $\exp \left(-j k_{y} y_{0}\right)$ based on the prior knowledge of a dispersion relation.
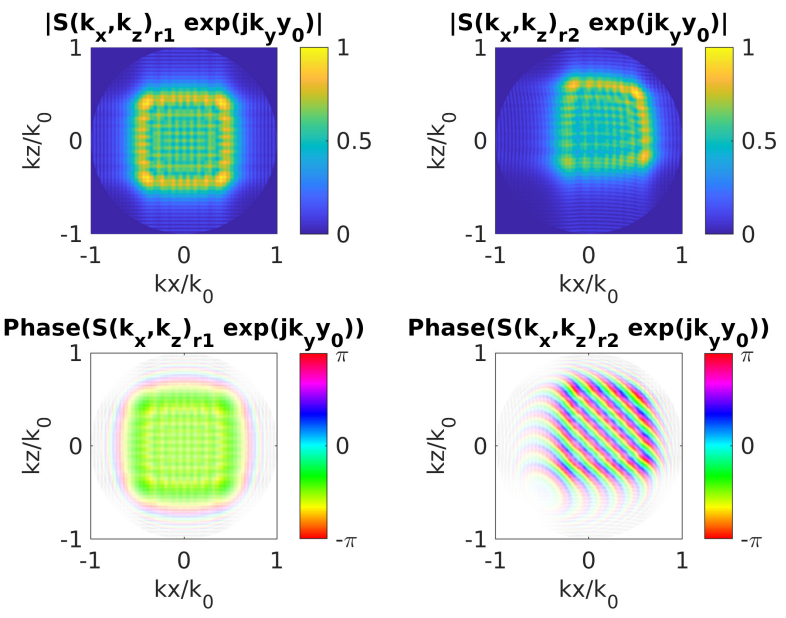

Fig. 5. Plane wave spectra are multiplied by the conjugate of the reference signal $\exp \left(-j k_{y} y_{0}\right)$. Note that the phase is almost constant when the antenna is located on the stationary phase point and that when the radiating element undergoes a translation in the $y=y_{0}$ plane, the calculation makes it possible to obtain a plane wave whose frequency and direction are directly related to the realized displacement.

The location of the radiating source can finally be computing with a 2-D inverse Fourier transform

$$
\hat{\sigma}(x, z)_{r_{i}}=\mathfrak{F}_{2 D}^{-1}\left(\int S\left(k_{x}, k_{z}\right)_{r_{i}} \exp \left(j k_{y} y_{0}\right) d f\right) .
$$

With this pointlike radiating element, the correlation between the plane wave spectrum of the measured signal and the reference signal having given rise to a phase distribution similar to a plane wave, and the final Fourier transform then converts this frequency information into the spatial position of the transmitting antenna (Fig. 5). As all these illustrations have been presented as part of a single-frequency measurement, it is only possible to resolve this element according to the transverse dimensions, parallel to the radiating aperture. The transmit antenna range can then be determined by performing a broadband measurement, summing the different frequency reconstructions in each plane in a coherent manner.

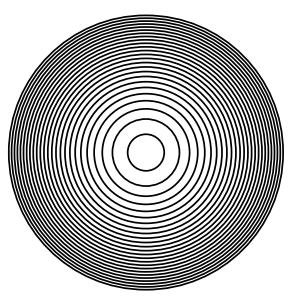

(a)

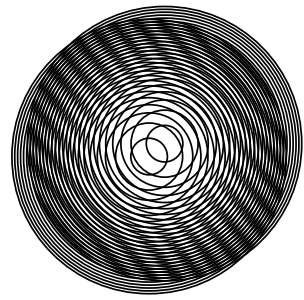

(b)
Fig. 6. Illustration of RMA properties using a Moiré pattern. This figure is obtained by superimposing two identical figures (a), one of which has undergone translation, forming a plane wave whose orientation and frequency are defined by this same translation (b). It is possible to print such shapes on both a white sheet and a transparent sheet in order to manipulate with more ease the two figures for the study of the formed plane wave.

For illustrative and pedagogical purposes, it is finally possible to reproduce the behavior of the complex correlation of $S\left(k_{x}, k_{z}\right)$ and $\exp \left(-j k_{y} y_{0}\right)$ by means of a simple pattern printed on both a white and a transparent sheet (Fig. 6). The superposition of these two drawings represented in the Fourier domain allows to create a plane wave by forming a Moiré pattern [24], [25].

To conclude this section, the RMA is a mathematical tool that is relatively simple to implement and very effective when combined with fast Fourier transforms. However, its mathematical foundations are complex and can largely benefit from more pragmatic analytical tools that allow us to understand the essence of the effectiveness of this technique, as well as its limitations. Although this principle is illustrated in the simplistic case of an array used in reception and associated with a source point in transmission, this principle can be directly transposed to more complex problems by adapting the dispersion relation. In the case of more complex targets, the superposition principle applied with the first-order Born approximation guarantees the correct functioning of this technique.

In light of the elements presented in this section, it is now possible to propose a technique for improving the implementation of RMA to MIMO systems. A matrix method is thus presented in Section III for replacing the delicate interpolation of the transverse projections of the $k$-vectors used in the conventional implementation of the MIMO RMA.

\section{Transverse Spectrum Deconvolution RANGe Migration Algorithm}

It is of interest in the first place to study the stationary phase method with particular attention, as this development is the keystone for treating the imaging problem in the plane wave domain. The complete mathematical development makes it possible to obtain the following expression, linking the measured signal, converted in the plane wave domain, to the reflectivity function of the target:

$$
S=\frac{1}{4 k_{y_{t}} k_{y_{r}}} \int_{r} \sigma(r) e^{-j k_{y} y} e^{-j k_{x} x} e^{-j k_{z} z} d^{3} r
$$

where the parameters $k_{y_{t}}, k_{y_{r}}, k_{x}, k_{y}$, and $k_{z}$ are the same as defined in (7)-(11). The complete calculation, which is 


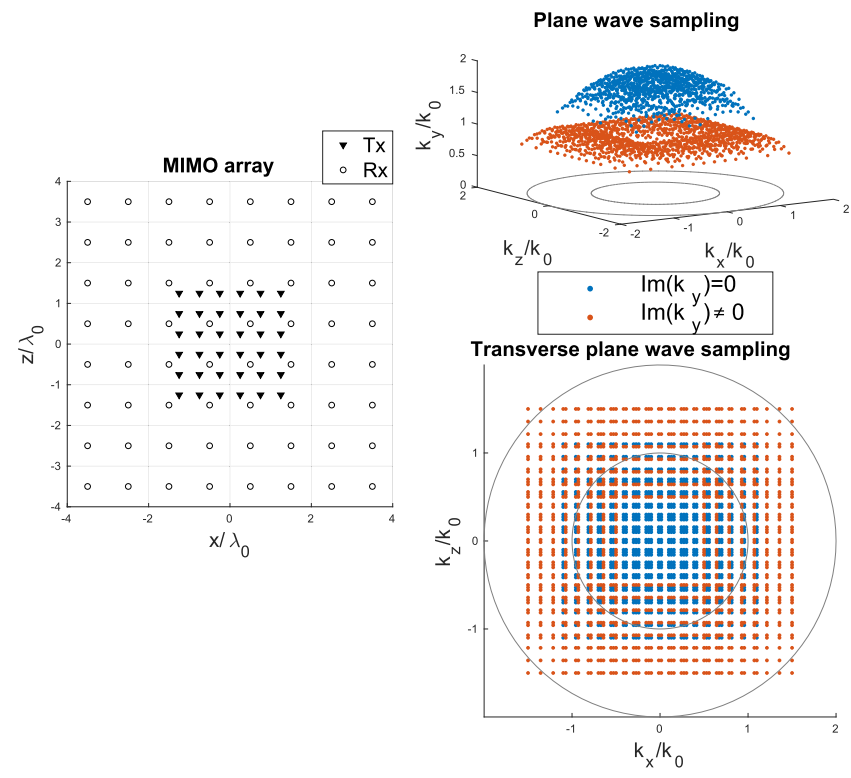

Fig. 7. Impact of spatial sampling of an MIMO imaging system on frequency components measured in the equivalent plane wave domain, represented at a single frequency $f_{0}$. The plane wave samples with a nonzero imaginary part are represented in a different color, and their magnitude tends to decrease rapidly with propagation, especially for components whose transverse projection is close to $2 k_{0}$.

relatively long and tedious, is presented in the Appendix in order to lighten this section. The resulting form is, up to a scalar constant, similar to the result given in (6) and [13]. This development highlights an important fact. It is necessary to preserve the $1 / R$ amplitude function to obtain a compact form as presented in (14).

\section{A. Principle of the RMA TSD}

Section II provided a visual interpretation of how RMA works, confirming the importance of having a reference function adapted to each antenna array architecture. The key element of this technique is the dispersion relation, the expression of which is obtained in the case of an MIMO architecture by asymptotic development of the integral expression of measured signals ( [13] and Appendix). This calculation also highlights the interaction between the transmitted and received plane waves, forming composite wave vectors described by (7)-(11). The most crucial part of the technique proposed in [13] corresponds to the interpolation step allowing the MIMO matrix $S\left(k_{x_{t}}, k_{z_{t}}, k_{x_{r}}, k_{z_{r}}, f\right)$ to be transformed into a 3-D matrix $S\left(k_{x}, k_{z}, f\right)$ expressed as a function of the new composite wave vectors. This transformation can be carried out using various interpolation techniques, which are particularly time-consuming and memory-intensive when applied to large MIMO systems. The sampling of the plane wave domain depends directly on that of the arrays used in transmission and reception. This principle is illustrated with an example in Fig. 7.

The transmit array is made of $n_{x_{t}} \times n_{z t}=6 \times 6$ elements, with a spacing of $d_{x_{t}}=d_{z_{t}}=0.5 \lambda_{0}$, and the receive array is made of $n_{x_{r}} \times n_{z_{r}}=8 \times 8$ elements, with a spacing of $d_{x_{r}}=$ $d_{z_{r}}=\lambda_{0}$. This spatial sampling directly defines the number



Fig. 8. Representation of the interaction between transverse components of transmitted and received plane wave spectra. The signals measured by the antennas and then converted in the $k$-space correspond to the signature of the scene to be imaged, interrogated by the interaction of transmit and receive spectra.

of transverse modes excited by each array, the combination of which may possibly be nonuniform, imposing additional interpolation steps slowing down the computation of images. It is proposed to simplify this step by developing a matrix technique to interrogate the transverse components of the spectrum of the scene to be imaged. This technique is first explained by means of an illustration (Fig. 8).

This technique is based on interrogating the reflection function of the target represented in the plane wave domain. Each element of the matrix $S\left(k_{x_{t}}, k_{z_{t}}, k_{x_{r}}, k_{z_{r}}, k\right)$ physically corresponds to the interaction between the radiation patterns generated in the desired independent directions (represented here by the transverse components of the wave vectors). The spatial limitations and sampling of the antenna arrays used for transmitting and receiving do not allow the interrogation of infinitely fine spectral lines. This approach then makes it possible to take into account, and even to exploit, the diversity of the radiated plane waves interacting with the unknown response of the target to be imaged. Considering radiation patterns $P_{t}\left(k_{x}, k_{z}\right)$ and $P_{r}\left(k_{x}, k_{z}\right)$ obtained by computing Fourier transforms of the array sampling functions and the analysis presented in Fig. 8, the expression of signals in the $k$-space is then written as the following convolution products:

$$
\begin{array}{r}
S=\int_{k_{x}} \int_{k_{z}} P_{t}\left(k_{x}-k_{x_{t}}, k_{z}-k_{z_{t}}\right) S_{c}\left(k_{x}, k_{z}, k\right) \\
P_{r}\left(k_{x}-k_{x_{r}}, k_{z}-k_{z_{r}}\right) d k_{x} d k_{z}
\end{array}
$$

where $S_{c}\left(k_{x}, k_{z}, k\right)$ is the remapped signal expression expressed in the $k$-space. If the samplings of the transmit and receive antenna arrays are separable functions, one can express 
the radiation patterns as

$$
\begin{aligned}
& P_{t}\left(k_{x}, k_{z}\right)=P_{t, x}\left(k_{x}\right) \cdot P_{t, z}\left(k_{z}\right) \\
& P_{r}\left(k_{x}, k_{z}\right)=P_{r, x}\left(k_{x}\right) \cdot P_{r, z}\left(k_{z}\right) .
\end{aligned}
$$

The different functions are then factorized so as to group together the same variables

$$
\begin{gathered}
P_{x}\left(k_{x}, k_{x_{t}}, k_{x_{r}}\right)=P_{t, x}\left(k_{x}-k_{x_{t}}\right) \cdot P_{r, x}\left(k_{x}-k_{x_{r}}\right) \\
P_{z}\left(k_{z}, k_{z_{t}}, k_{z_{r}}\right)=P_{t, z}\left(k_{z}-k_{z_{t}}\right) \cdot P_{r, z}\left(k_{z}-k_{z_{r}}\right)
\end{gathered}
$$

leading to the simplified expression of $S$

$$
\begin{array}{r}
S=\int_{k_{x}} \int_{k_{z}} P_{x}\left(k_{x}, k_{x_{t}}, k_{x_{r}}\right) P_{z}\left(k_{z}, k_{z_{t}}, k_{z_{r}}\right) \\
S_{c}\left(k_{x}, k_{z}, k\right) d k_{x} d k_{z}
\end{array}
$$

It is then possible to write a matrix formalism to express the link of $S_{c}$ and $S$ for each wavenumber $k$, with the help of the following matrices $\bar{S}_{c}(k) \in \mathbb{C}^{n_{k_{x}} \times n_{k_{z}}}$,

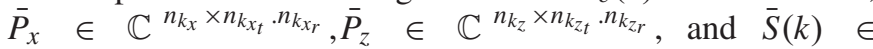

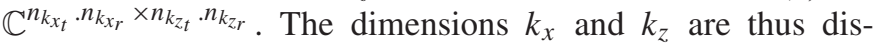
cretized in uniformly sampled vector, defining the maximum extension of the imaging domain. Equation (20) is then expressed as

$$
\bar{S}(k)=\bar{P}_{x} \bar{S}_{c}(k) \bar{P}_{z} .
$$

The expression of $\hat{\bar{S}_{c}}(k)$ for each value of $k$ thus takes the following form:

$$
\hat{\bar{S}}_{c}(k)=\bar{P}_{x}^{+} \bar{S}(k) \bar{P}_{z}^{+}
$$

where the symbol ${ }^{+}$stands for the pseudoinverse operator. This approach, referred as transervse spectrum deconvolution (TSD), has multiple advantages compared with conventional techniques based on the interpolation of transverse wave vectors.

1) This transformation does not depend on the sampling of transverse wave vectors and therefore does not require signal preconditioning.

2) The transfer matrices $\bar{P}_{x}$ and $\bar{P}_{z}$ do not depend on frequency or wavenumber. This greatly reduces the memory and time consumption of the algorithm, which requires only a single calculation of the pseudoinverse of small matrices with respect to those of the $S$ signal. This approach makes computing highly parallelizable and particularly suitable for array programming languages.

3) This formalism remains fully compatible with the use of nonuniform antenna arrays that remains separable functions. A precalculation of the $\bar{P}_{x}^{+}$and $\bar{P}_{z}^{+}$matrices or the use of nonuniform fast Fourier transform nuFFT) can speed up digital processing, without requiring the use of interpolation of the associated signals.

4) This approach makes it possible to consider the interaction of the scene with all the transmit and receive plane waves emitted for each beam formation, exploiting all the secondary lobes which usually represent elements that are detrimental to image quality.

5) The dimensions of the scene to be imaged are directly defined by sampling the vectors $k_{x}$ and $k_{z}$ on which the radiation patterns are calculated, avoiding the use of zero padding on 5-D matrices that already represent a certain constraint on memory consumption.

This last step of the calculation finally made it possible to move from a 5-D signal $S\left(k_{x_{t}}, k_{z_{t}}, k_{x_{r}}, k_{z_{r}}, k\right)$ to a remapped signal with only three uniformly sampled dimensions $S_{c}\left(k_{x}, k_{z}, k\right)$. In accordance with conventional approaches, it is then possible to use the dispersion relation determined using the asymptotic calculations presented in the Appendix to express the third dimension of $S_{c}$ as a function of the longitudinal projection of the wave vector $k_{y}$ instead of the wavenumber $k$. The expression $k_{y}$ is given in the following:

$$
k_{y}=\sqrt{k^{2}-k_{x_{t}}^{2}-k_{z_{t}}^{2}}+\sqrt{k^{2}-k_{x_{r}}^{2}-k_{z_{r}}^{2}} .
$$

The plane wave components having been expressed according to the same vectors $k_{x}$ and $k_{z}$, it is then possible to obtain a simplified expression of $k_{y}$

$$
k_{y}=2 \sqrt{k^{2}-k_{x}^{2}-k_{z}^{2}} .
$$

The reflectivity function of the target can finally be estimated

$$
\begin{aligned}
\hat{\sigma}_{\mathrm{tsd}}(x, y, z)=\int_{k_{x}} \int_{k_{z}} \int_{k} 4 k_{y}^{2} S_{c}\left(k_{x}, k_{z}, k\right) \\
e^{j\left(k_{x} x+k_{z} z+k_{y} y\right)} d k d k_{x} d k_{z}
\end{aligned}
$$

The numerical computation of this expression can still be quite slow when working with large antenna arrays due to the nonuniform sampling of the matrix $k_{y}$ preventing from implementing fast Fourier transforms. This problem can be alleviated by using the Solt interpolation, consisting in resampling the matrix $S_{c}\left(k_{x}, k_{z}, k\right)$ over uniform grids fitting a uniformly sampled vector $k_{y}$, leading to $S_{c i}\left(k_{x}, k_{z}, k_{y}\right)$. The association of the proposed technique with the Stolt interpolation is referred to as TSDi in this paper. Finally, the reflectivity function can be estimated using a 3-D inverse fast Fourier transform

$$
\hat{\sigma}_{\text {tsdi }}(x, y, z)=\mathcal{F}_{3 D}^{-1}\left(S_{c i}\left(k_{x}, k_{z}, k_{y}\right)\right) .
$$

The different algorithms studied in this paper are summarized in the form of a diagram presented in Fig. 9.

A new implementation of RMA adapted to MIMO systems has been proposed. The numerical and experimental validations of this technique are presented in Section III-B in order to highlight the advantages and disadvantages of this approach compared with the existing techniques.

\section{B. Numerical Validation}

A first validation is carried out using a numerical simulation to measure the interaction of antenna arrays with target points. Such an approach will make it possible to highlight the computing times, memory consumption, and spatial resolutions obtained in the case of the application of RMA as defined in [13] and using the approach proposed in this paper. The system proposed for the study of these algorithms consists of two monostatic arrays (identical and sharing the same coordinates) used in transmission and reception. The simulation is carried out in the K-band (18-26 GHz) using an antenna spacing of $0.8 c / f_{\max }=9.2 \mathrm{~mm}$ in the transverse 


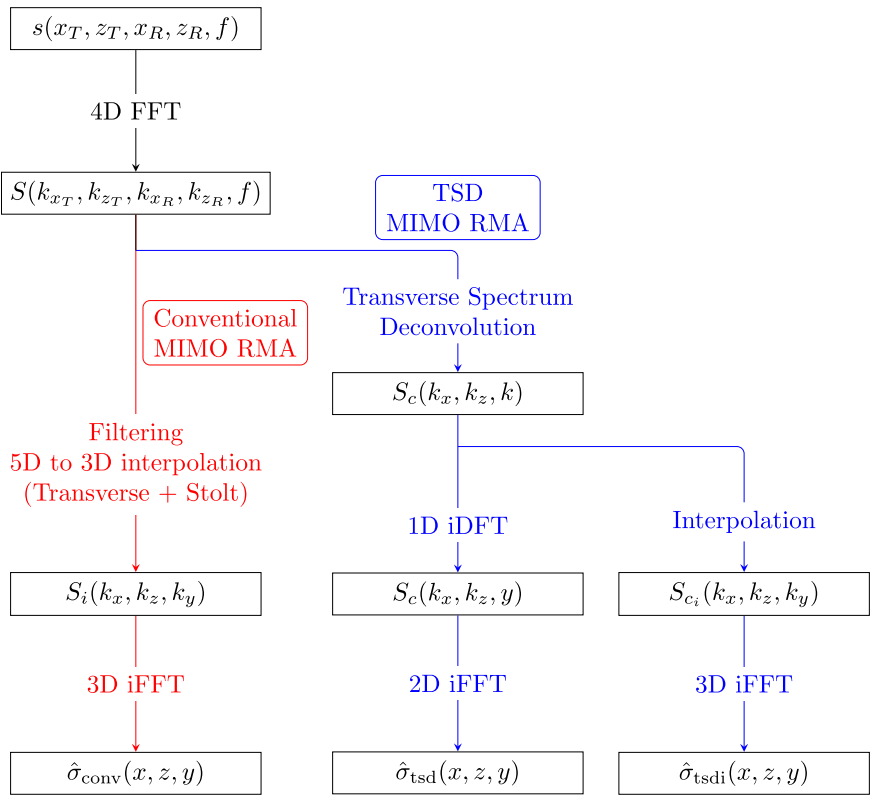

Fig. 9. Comparison of the conventional MIMO RMA algorithm with the proposed TSD method. It is possible to implement this approach with a DFT or to speed up its execution with Stolt trilinear interpolation.

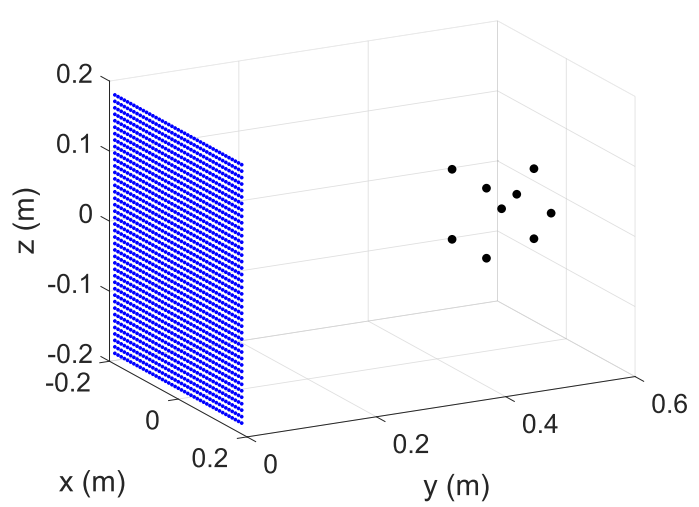

Fig. 10. Illustration of the proposed MIMO imaging simulation for the validation of this new imaging technique. An array of $40 \times 40$ antennas used in both transmission and reception is used to reconstruct the image of these nine source points.

directions $x$ and $z$. The arrays are placed in the plane $y=0$, while nine target points are arranged around a distance of $y=0.5 \mathrm{~m}$ (Fig. 10). Target spacing is chosen so as to draw a square of $3 \times 3$ elements separated by $5 \mathrm{~cm}$ in transverse dimensions. The four targets located on the corners of the square are placed at $y=0.045 \mathrm{~m}$, the central element at $y=$ $0.5 \mathrm{~m}$, and the four elements remaining at $y=0.55 \mathrm{~m}$. For this first simulation, antenna arrays are made up of $n_{b a}=40$ antennas per side, corresponding to the interaction between $n_{b a}^{4}=2.56$ million elements measured by this MIMO system.

Interaction is measured between transmitters and receivers for each of the 20 frequency points uniformly sampling the $18-26-\mathrm{GHz}$ operating bandwidth. This matrix is then used to calculate an image using the conventional implementation of the RMA MIMO algorithm proposed in [13]. The result of the implementation of this first approach is shown in Fig. 11.

It can be seen that the reconstructed image has an uneven amplitude distribution with an energy concentration on the

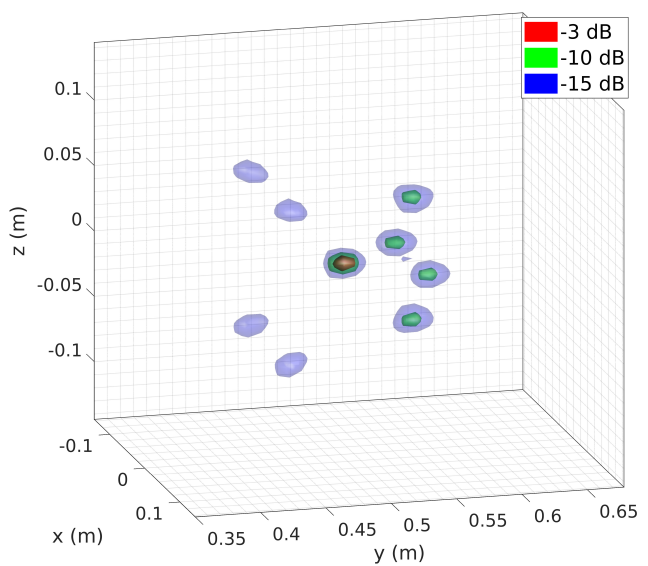

Fig. 11. Implementation of the conventional MIMO RMA algorithm. Isosurfaces of the normalized image are represented for values of $-3,-10$, and $-15 \mathrm{~dB}$.

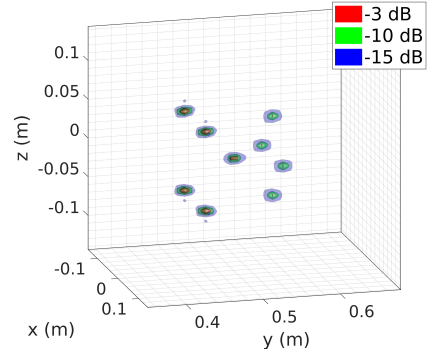

(a)

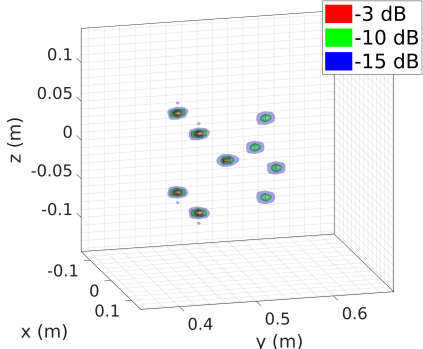

(b)
Fig. 12. Implementation of the proposed method. (a) $\hat{\sigma}_{\text {tsd }}-$ full computation of the Fourier transform using the dispersion relation. (b) $\hat{\sigma}_{\text {tsdi }}$-accelerated method based on a Stolt trilinear interpolation. Isosurfaces of the normalized image are represented for the values of $-3,-10$, and $-15 \mathrm{~dB}$.

central target (used as a stationary phase point) and on the four points furthest away from the array. It is necessary to specify at this stage that the raw matrix is used and zero padding has not been implemented. For comparison purposes, the same matrix is again used for the implementation of the proposed method. For this first example, the pseudoinverses of the radiation matrices $\bar{P}_{x}$ and $\bar{P}_{z}$ are simply calculated by using matched filtering, such as $\bar{P}_{x}^{+}=\bar{P}_{x}^{H}$ and $\bar{P}_{z}^{+}=\bar{P}_{z}^{H}$, where ${ }^{H}$ is the conjugate-transpose operator. The results are presented in Fig. 12.

In this configuration and despite the absence of zero padding, the amplitude distribution appears more uniform across the space. This difference is directly related to the use of interpolation techniques in the conventional approach, allowing to merge the different transverse wave vectors, which can introduce distortions in the reconstructed images. In order to highlight these differences, two transverse views of the reconstructed images $\hat{\sigma}_{\text {conv }}$ and $\hat{\sigma}_{\text {tsd }}$ are presented in Fig. 13.

It can be observed that the position of the targets on the corners undergoes a small translation from their real locations in the case of the conventional approach, where the proposed technique allows a correct estimation of the positions of the targets, forming a square of $3 \times 3$ elements separated by $5 \mathrm{~cm}$ along $\mathrm{x}$ and $\mathrm{z}$. The proposed approach thus offers a better 


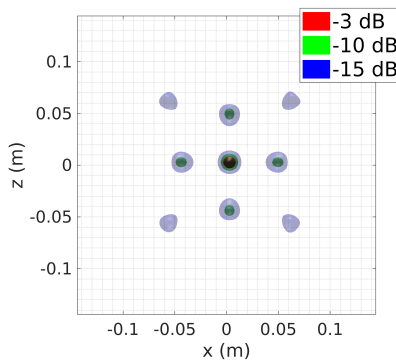

(a)

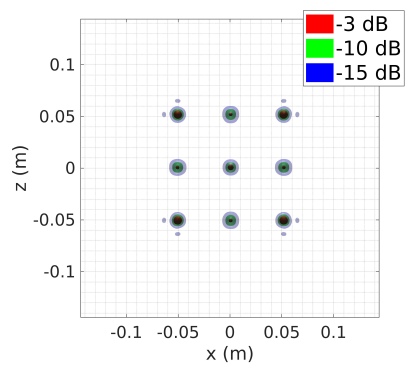

(b)
Fig. 13. Transverse views of reconstructed images in the case of (a) classical RMA MIMO implementation and (b) proposed technique.



(a)



(b)
Fig. 14. Comparison of (a) cross-sectional and (b) longitudinal resolutions obtained in the imaging of a source point placed in $(x, y, z)=(0,0.05,0)$. The curves along the $\mathrm{x}$ - and $\mathrm{z}$-axis are similar because of the antenna array symmetry.

estimation of the transverse spectrum of the scene to be imaged by using a deconvolution-based technique exploiting the entire spectrum of transmission and reception spectra. The transverse and longitudinal resolutions are then compared using the same system in the framework of the reconstruction of a simple source point placed at $(x, y, z)=(0,0.0 .5,0) \mathrm{m}$. The results obtained are presented in Fig. 14.

The midheight transverse resolution measured for the proposed technique is $7.6 \mathrm{~mm}$, compared to $12.1 \mathrm{~mm}$ for the conventional MIMO RMA. The theoretical transverse resolution can be approximated as $\delta_{x, z} \approx \lambda_{\min } R /\left(2 L_{x, z}\right)=$ $7.6 \mathrm{~mm}$ [13], where $L_{x, z}$ are the aperture dimensions along the $x$ and $z$ directions, and is in good agreement with the results obtained with the proposed method. In the case of depth resolution, $12.8 \mathrm{~mm}$ is obtained for the proposed approach implemented with a direct Fourier transform (DFT), $17.8 \mathrm{~mm}$ for the TSD associated with an interpolation step and a fast Fourier transform, and $19 \mathrm{~mm}$ in the case of conventional technique. A model based on the bandwidth $B$ is usually considered for estimating the range resolution, as $\delta_{y} \approx c / 2 B=18.7 \mathrm{~mm}$. This result is in good agreement with the resolution obtained with the conventional method. Considering the shells formed in the $k$-space represented in Fig. 7, the upper bound of the range resolution can be defined as $\delta_{y}=2 \pi /\left(2 k_{\max }\right)=c / f_{\max }=11.5 \mathrm{~mm}$. The resolution obtained by applying the proposed technique is thus closer to this theoretical limit by an improved exploitation of the frequency components of the measured signals.

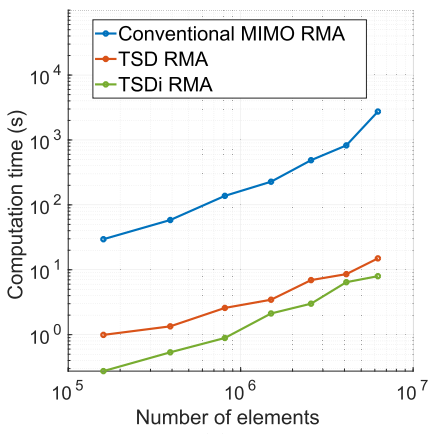

(a)

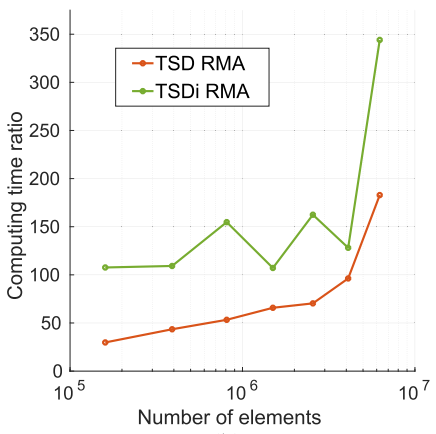

(b)
Fig. 15. (a) Comparison and (b) ratio of the computation times of the conventional RMA MIMO algorithm and the proposed method according to the number of equivalent radiating elements.

The performance of these three algorithms is now compared. The matrix technique proposed in this paper has the advantage of reducing the memory required for the proper functioning of the program, as well as the associated computational times, especially when the number of radiating elements of the considered arrays is important. A comparison of the image computing time is thus proposed, varying the number of antennas used in transmission and reception arrays. To limit the number of variables in this study, we are still using a monostatic system, made up of a square array used for both transmission and reception, with the number of antennas per side denoted $n_{b a}$. This study is carried out for $n_{b a}$ ranging from 10 to 40 antennas per side of the arrays, leading to matrices of $n_{b a}^{4}$ elements for each of the 20 frequency points of the operating K-band. The calculation times are thus compared for the processing of 10000-2.56 million equivalent radiating elements, obtained by combining all pairs of transceivers. The calculations are carried out with MATLAB, using a computer equipped with 16 GB of RAM and a six-core 3.5-GHz CPU. The results of this study are presented in Fig. 15.

The substitution of the interpolation of transverse spectral components by fusion achieved with a matrix technique not only optimizes the accuracy and resolution of reconstructed images but also allows for faster image computation up to a ratio of 75 for the largest case computed with $40^{4}$ equivalent radiating elements.

To conclude this study, a more realistic scenario is investigated, simulating the interaction with a mannequin carrying a firearm at the belt level, imported from an STL file. The simulation is carried out according to the numerical models described in [26] and [27] by computing the electric fields radiated by a set of magnetic dipoles in the target space. This choice is justified in metasurface-based computational imaging applications to convert measured or analytically derived tangential electric fields into secondary sources injected in the numerical solver. The target under consideration consists of 67455 facets chosen small enough with respect to the dimensions of the radiating aperture to be able to apply a physical optics approximation [28].

The contribution of each facet is thus calculated according to its orientation and dimension following a square cosine model. The qualitative validation of such an approach with 


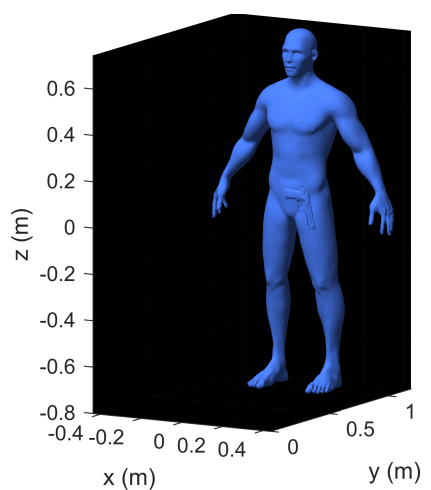

(a)



(b)
Fig. 16. Simulation of a faceted 3-D object. (a) Imported human body carrying a firearm. (b) Interaction between each antenna of the $71 \times 71$ MIMO array represented in red and the facets of the target is calculated.

experimental results can be found in [26] and [27]. A simulation is carried out considering a 2-D MIMO antenna array formed by $71 \times 71$ elements, in which each antenna element operates in the monostatic mode, with a spatial sampling of $0.7 \lambda_{\min }=6.92 \mathrm{~mm}$ and placed at $1 \mathrm{~m}$ from the target (Fig. 16). These simulations are carried out in the frequency domain over 18-26-GHz frequency uniformly sampled by 48 points (Fig. 17).

Before analyzing the simulation results, it is important to identify the approximations that were considered for their computation. These simulations are based on the first Born approximation and do not take into account multipath phenomena. In an experimental application and under unfavorable body postures, it may be possible to observe artifacts caused by these multiple bounces, particularly between the legs and under the arms of the mannequin. However, the use of polarimetric information in such a context [17] could help to identify and filter these detrimental effects [29].

The simulated mannequin is made of metal facets with a reflection coefficient of 0.8 to match the average reflectivity of a human body [27]. Any depolarization or delay phenomenon related to the dielectric nature of a real human body is therefore not taken into account in this simulation [30]. As the impact of additive noise is rarely negligible for this type of application, it is also proposed to illustrate the impact of this effect on reconstruction under relatively unfavorable conditions. A signal-to-noise ratio (SNR) of $3 \mathrm{~dB}$ is thus considered for this study. This SNR is calculated from the energy of the strongest signal, determining the variance of the zero mean Gaussian noises added to all the measurements.

The volumetric reconstructions presented correspond to isosurfaces extracted at $-6 \mathrm{~dB}$ from each image, previously normalized in amplitude. The color code corresponds to the depth of the reconstructed surfaces, allowing the presence of the firearm to be identified, as well as the position in space of the limbs of the target. These visualizations are simplified by representing only a 2-D view of the reconstructed information, keeping only the elements with the strongest linear magnitude as a function of depth, corresponding to the right-hand images of Fig. 17. One might notice that the trilinear interpolation
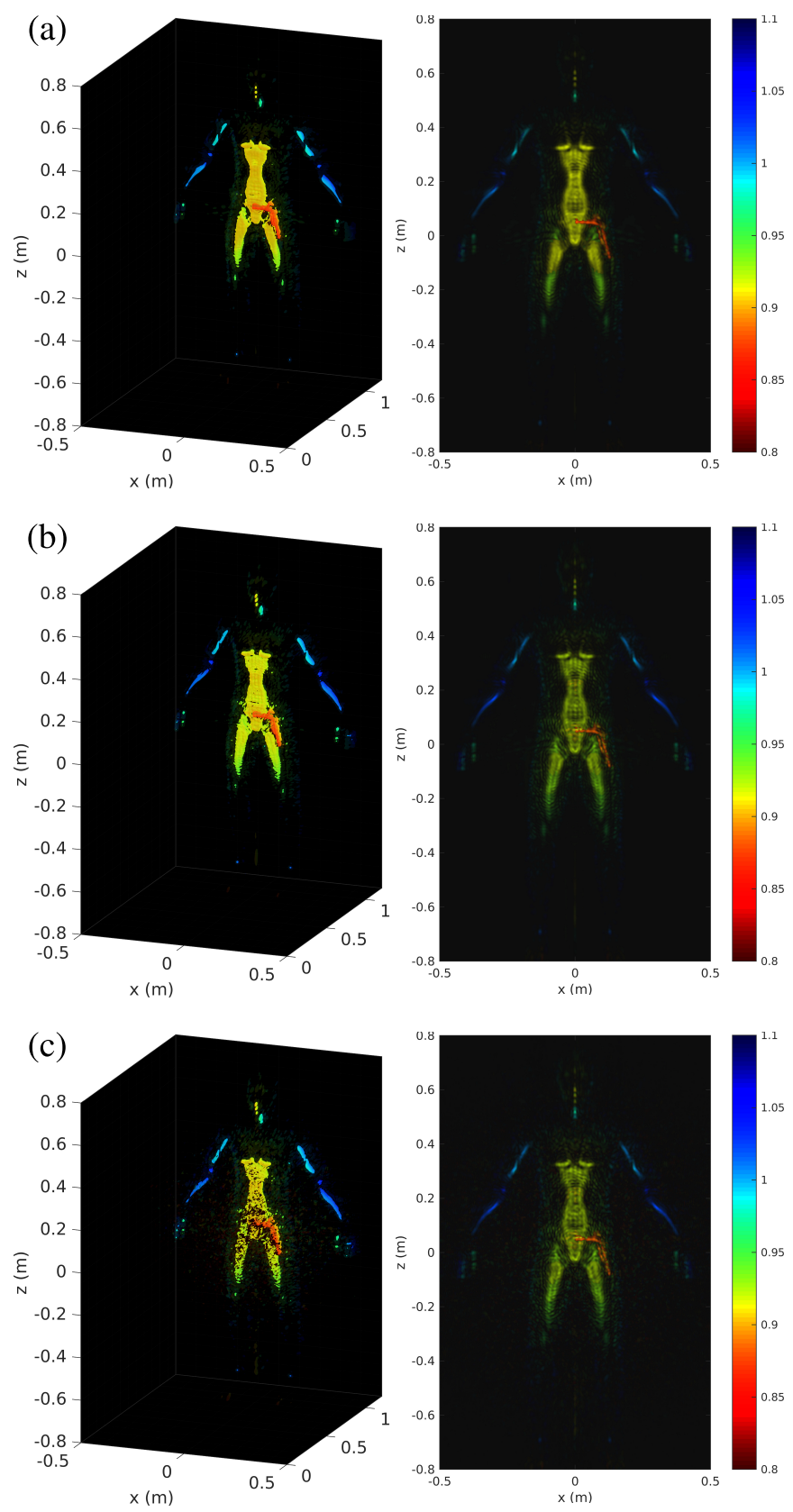

Fig. 17. (a) Images obtained from the TSD method from a $71 \times 71$ MIMO antenna array. (b) Images reconstructed with the TSDi approach based on Stolt interpolation under the same conditions. (c) Reconstructions by TSDi using the same simulated signals, this time with an SNR of $3 \mathrm{~dB}$ to illustrate the impact of additive noise.

step used for the TSDi technique adds distortions compared with the results obtained by simple TSD. It would be possible to limit the occurrence of such distortions by using more advanced interpolation techniques, but their interest would quickly be questionable if the associated calculation times are examined. The addition of Gaussian noise to the signals has a clearly visible impact on the volumetric reconstruction of the image by TSDi compared with the results obtained for an infinite SNR. The extraction of the isosurface is affected by the presence of a speckle making it a little more difficult to identify the object carried by the target. The depth information 
TABLE I

Computation Times of the Images PREsented in Fig. 17

\begin{tabular}{|c|c|c|}
\hline & Total (s) & FFTs only (s) \\
\hline $71 \times 71$ array - TSD RMA & 27.1 & 15 \\
\hline $71 \times 71$ array - TSDi RMA & 24.8 & 15.1 \\
\hline
\end{tabular}

extraction method chosen for the 2-D display, however, makes it possible to obtain a visualization of the target much less impacted by the noise added to the measurements. Despite the particularly unfavorable conditions simulated here with an SNR of $3 \mathrm{~dB}$, the reconstruction algorithm is relatively robust to noise by exploiting the spatial averaging of the very large number of signals coherently summed in the target space (see [19, Sec. 4.6]).

All these images were calculated on the same computing server with a double CPU with 10 cores running at $2.4 \mathrm{GHz}$ and equipped with $128 \mathrm{~GB}$ of RAM, allowing the processing of all data with the same machine and a comparison of computing times gathered in Table I, including, for each execution, the computation times only devoted to fast Fourier transforms.

It should be noted that the difference in computation times between TSD RMA and TSDi RMA eventually diminishes with the significant increase in the size of the matrices to be processed due to a faster growth in the complexity of trilinear interpolation computation compared with the initial approach based on the matrix computation of the Fourier transform. It is also important to note that the computation time devoted solely to fast Fourier transforms represents a growing fraction of the total computation time of this approach, exceeding 50\% for the array of $71 \times 71$ elements.

These different reconstructions allow us to conclude that it is possible to adapt the antenna array architecture and the computing method according to the needs of each application in order to optimize the resolution and computing time of reconstructed images.

\section{CONCLUSION}

An imaging technique applied to MIMO imaging systems was presented in this paper. This new breakthrough is intended to overcome memory and computational time limitations imposed by conventional imaging techniques while not sacrificing the accuracy and resolution of estimations. A review of all the elements necessary to understand MIMO Fourier processing techniques was proposed, identifying the crucial points necessary for an intuitive presentation of this complex technique based on asymptotic developments of complex integrals. It was then possible at this stage to propose a new reconstruction method in the field of plane waves based on a physical interpretation of the interaction between the radiation of the imaging system and different targets. A numerical study revealed significant time savings in computation between the proposed method and the conventional approach proposed [13], particularly when imaging systems include a large number of antennas and large volumes to be imaged. Although these antenna array architectures are not commonly used for the usual MIMO imager implementations as they would be very expensive and would present a significant redundancy of measured information, new implementations based on the use of various frequency-diverse antennas directly face these challenges. Further studies will be carried out in the future to identify possible new optimizations of this technique. Fast Fourier transforms now represent the largest fraction of the computational time of this approach, so it will be possible to accelerate these processes by adapting these computations on field-programmable gate array (FPGA) or GPU instead of the simple multicore CPU used for this proof of principle [31]. It will also be possible to parallelize the reconstruction of radar images by separating them into a set of subdomains centered around different stationary phase points following the technique presented in [32], allowing at the same time to reduce the distortions applied to the elements reconstructed far from the stationary phase point.

\section{APPENDIX}

In this section, it is proposed to study, in a comprehensive manner, the asymptotic development of the integral equation of the signals received by an MIMO array using the stationary phase method. In addition to the pedagogical value of this development when combined with the intuitive illustrations given in Figs. 4-6, it makes it possible to highlight a certain number of calculation steps that are eluded in [13] for compactness concerns. A fraction of these developments can be found in [12] and [33], although initially applied to a synthetic aperture radar.

To the best of our knowledge, all asymptotic developments proposed in this field are based on a simplified derivation of the measured signals, considering that amplitude terms have a negligible usefulness in comparison with phase terms and can thus be removed. It is shown in this section that it is necessary to keep the decay term $1 / R$, evaluated at the stationary phase point so that the final expression converges toward a form very close to that given in the references mentioned earlier. From a physical point of view, the conservation of the amplitude term also seems justified insofar as this information remains of particular interest in the context of imaging applications in the Fresnel zone.

We start the calculations from the initial formalism of the MIMO signal simplified according to a scalar field approximation and to the first-order Born approximation

$$
s\left(x_{t}, z_{t}, x_{r}, z_{r}, k\right)=\int_{r} \frac{\sigma(r)}{16 \pi^{2}} \frac{e^{-j k R_{t}}}{R_{t}} \frac{e^{-j k R_{r}}}{R_{r}} d^{3} r
$$

with

$$
\begin{aligned}
R_{t} & =\sqrt{\left(x-x_{t}\right)^{2}+y^{2}+\left(z-z_{t}\right)^{2}} \\
R_{r} & =\sqrt{\left(x-x_{r}\right)^{2}+y^{2}+\left(z-z_{r}\right)^{2}} \\
R & =\sqrt{x^{2}+y^{2}+z^{2}} \\
d V & =\partial x \partial y \partial z .
\end{aligned}
$$

The spatial dimensions are expressed in the Fourier domain in order to consider the interaction between the emitted and received plane waves and the target to be imaged

$$
S\left(k_{x_{t}}, k_{z_{t}}, k_{x_{r}}, k_{z_{r}}, k\right)=\mathfrak{F}_{4 D}\left(s\left(x_{t}, z_{t}, x_{r}, z_{r}, k\right)\right) .
$$


The development of the expression of this signal makes it possible to factorize the transmission and reception terms

$$
\begin{aligned}
S= & \int_{r} \int_{A_{t}} \int_{A_{r}} \frac{\sigma(r)}{16 \pi^{2}} \frac{e^{-j k R_{t}}}{R_{t}} \frac{e^{-j k R_{r}}}{R_{r}} \\
& e^{-} j k_{x_{t}} x_{t} e^{-} j k_{z t} z_{t} e^{-} j k_{x_{r}} x_{r} e^{-} j k_{z_{r}} z_{r} d A_{r} d A_{t} d^{3} r \\
= & \int_{r} \frac{\sigma(r)}{16 \pi^{2}}[\underbrace{\int_{A_{t}} \frac{e^{-j k R_{t}}}{R_{t}} e^{-j k_{x_{t}} x_{t}} e^{-j k_{z t} z_{t}} d A_{t}}_{E_{t}}] \\
& {[\underbrace{\int_{A_{r}} \frac{e^{-j k R_{r}}}{R_{r}} e^{-j k_{x_{r}} x_{r}} e^{-j k_{z r} z_{r}} d A_{r}}_{E_{r}}] d^{3} r }
\end{aligned}
$$

where the surface elements of the transmit and receive apertures are $d A_{t}=\partial x_{t} \partial z_{t}$ and $d A_{r}=\partial x_{r} \partial z_{r}$, respectively. The integrals $E_{t}$ and $E_{r}$ share the same mathematical form that can be simplified using the method of stationary phase. These expressions are developed here with a generic index $i$ standing for $t$ or $r$

$$
E_{i}\left(k_{x_{i}}, k_{z_{i}}, k\right)=\int x_{i} \int z_{i} \frac{e^{-j k R_{i}}}{R_{i}} e^{-j k_{x_{i}} x_{i}} e^{-j k_{z_{i}} z_{i}} \partial x_{i} \partial z_{i} .
$$

The evaluation of this integral is carried out using asymptotic development. It is therefore necessary to express this expression in a particular oscillatory integral form

$$
\begin{array}{r}
E_{i}\left(k_{x_{i}}, k_{z_{i}}, k\right)=\int_{x_{i}} \int_{z_{i}} \frac{e^{-j k \sqrt{\left(x_{i}-x\right)^{2}+y^{2}+\left(z_{i}-z\right)^{2}}}}{\sqrt{\left(x_{i}-x\right)^{2}+y^{2}+\left(z_{i}-z\right)^{2}}} \\
e^{-j k_{x_{i}} x_{i}} e^{-j k_{z_{i}} z_{i}} \partial x_{i} \partial z_{i} \\
=\int_{x_{i}} \int_{z_{i}} \frac{1}{R} e^{j k \Phi} \partial x_{i} \partial z_{i}
\end{array}
$$

with

$$
\begin{aligned}
& \Phi=-\sqrt{\left(x_{i}-x\right)^{2}+y^{2}+\left(z_{i}-z\right)^{2}}-\frac{k_{x_{i}}}{k} x_{i}-\frac{k_{z_{i}}}{k} z_{i} \\
& R=\sqrt{\left(x_{i}-x\right)^{2}+y^{2}+\left(z_{i}-z\right)^{2}} .
\end{aligned}
$$

The factorization of the wavenumber $k$ makes it possible to introduce a phase term $\Phi$ that varies slowly with respect to the frequency. This development makes it possible to realize an asymptotic expansion of the integral, considering that the most significant contributions arises a saddle point called the stationary phase point $\left(x_{s}, z_{s}\right)$, and defined as

$$
\begin{aligned}
& \left.\frac{\partial \Phi}{\partial x_{i}}\right|_{x_{s}, z_{s}}=0 \\
& \left.\frac{\partial \Phi}{\partial z_{i}}\right|_{x_{s}, z_{s}}=0 .
\end{aligned}
$$

A second-order 2-D Taylor expansion of $\Phi$ is calculated at the stationary phase point $\left(x_{i}=x_{s}, z_{i}=z_{s}\right)$

$$
\begin{aligned}
\Phi \approx & \Phi\left(x_{s}, z_{s}\right)+\overbrace{\left.\frac{\partial \Phi}{\partial x_{i}}\right|_{x_{s}, z_{s}}}^{=0}\left(x_{i}-x_{s}\right)+\overbrace{\left.\frac{\partial \Phi}{\partial z_{i}}\right|_{x_{s}, z_{s}}}^{=0}\left(z_{i}-z_{s}\right) \\
& +\left.\frac{\partial^{2} \Phi}{\partial x_{i}^{2}}\right|_{x_{s}, z_{s}} \frac{\left(x_{i}-x_{s}\right)^{2}}{2 !}+\left.\frac{\partial^{2} \Phi}{\partial z_{i}^{2}}\right|_{x_{s}, z_{s}} \frac{\left(z_{i}-z_{s}\right)^{2}}{2 !} \\
& +\left.\frac{1}{2 !} \frac{\partial^{2} \Phi}{\partial x_{i} \partial z_{i}}\right|_{x_{s}, z_{s}}\left(x_{i}-x_{s}\right)\left(z_{i}-z_{s}\right) \\
& +\left.\frac{1}{2 !} \frac{\partial^{2} \Phi}{\partial z_{i} \partial x_{i}}\right|_{x_{s}, z_{s}}\left(x_{i}-x_{s}\right)\left(z_{i}-z_{s}\right) \\
\Phi & \Phi\left(x_{s}, z_{s}\right)+\left.\frac{\partial^{2} \Phi}{\partial x_{i}^{2}}\right|_{x_{s}, z_{s}} \frac{\left(x_{i}-x_{s}\right)^{2}}{2 !} \\
& +\left.\frac{\partial^{2} \Phi}{\partial z_{i}^{2}}\right|_{x_{s}, z_{s}} \frac{\left(z_{i}-z_{s}\right)^{2}}{2 !} \\
& +\left.\frac{\partial^{2} \Phi}{\partial x_{i} \partial z_{i}}\right|_{x_{s}, z_{s}}\left(x_{i}-x_{s}\right)\left(z_{i}-z_{s}\right) .
\end{aligned}
$$

The expression of $x_{s}$ and $z_{s}$ can first be obtained from the first derivatives vanishing at the stationary phase point

$$
\begin{aligned}
& \left.\frac{\partial \Phi}{\partial x_{i}}\right|_{x_{s}, z_{s}}=-\frac{x_{s}-x}{\sqrt{\left(x_{s}-x\right)^{2}+y^{2}+\left(z_{s}-z\right)^{2}}}-\frac{k_{x_{i}}}{k}=0 \\
& \left.\frac{\partial \Phi}{\partial z_{i}}\right|_{x_{s}, z_{s}}=-\frac{z_{s}-z}{\sqrt{\left(x_{s}-x\right)^{2}+y^{2}+\left(z_{s}-z\right)^{2}}}-\frac{k_{z_{i}}}{k}=0 .
\end{aligned}
$$

Equations (44) and (45) then lead to the following coupled equations:

$$
\begin{aligned}
& \left(x_{s}-x\right)^{2}=\frac{k_{x_{i}}^{2}}{k^{2}-k_{x_{i}}^{2}}\left(y^{2}+\left(z_{s}-z\right)^{2}\right) \\
& \left(z_{s}-z\right)^{2}=\frac{k_{z_{i}}^{2}}{k^{2}-k_{z_{i}}^{2}}\left(y^{2}+\left(x_{s}-x\right)^{2}\right) .
\end{aligned}
$$

The resolution of this last equation system makes it possible to determine the expression of the coordinates of the stationary phase point, extracting the positive roots for each case

$$
\begin{aligned}
& x_{s}=x+y \frac{k_{x_{i}}}{\sqrt{k^{2}-k_{x_{i}}^{2}-k_{z_{i}}^{2}}} \\
& z_{s}=z+y \frac{k_{z_{i}}}{\sqrt{k^{2}-k_{x_{i}}^{2}-k_{z_{i}}^{2}}} .
\end{aligned}
$$

The second derivatives $\partial^{2} \Phi / \partial x_{i}^{2}, \quad \partial^{2} \Phi / \partial z_{i}^{2}, \quad$ and $\partial^{2} \Phi / \partial x_{i} \partial z_{i}$ can now be evaluated, reminding that $\Phi=$ $-\left(\left(x_{i}-x\right)^{2}+y^{2}+\left(z_{i}-z\right)^{2}\right)^{(1 / 2)}-\left(k_{x_{i}} / k\right) x_{i}-\left(k_{z_{i}} / k\right) z_{i}$

$$
\begin{aligned}
\frac{\partial^{2} \Phi}{\partial x_{i}^{2}} & =\frac{\partial}{\partial x_{i}}\left(-\frac{x_{i}-x}{\sqrt{\left(x_{i}-x\right)^{2}+y^{2}+\left(z_{i}-z\right)^{2}}}-\frac{k_{x_{i}}}{k}\right) \\
& =\frac{y^{2}+\left(z_{i}-z\right)^{2}}{\left(\left(x_{i}-x\right)^{2}+y^{2}+\left(z_{i}-z\right)^{2}\right)^{\frac{3}{2}}} .
\end{aligned}
$$


Similarly, we evaluate the second derivative of the phase term according to $z_{i}$

$$
\frac{\partial^{2} \Phi}{\partial z_{i}^{2}}=-\frac{y^{2}+\left(x_{i}-x\right)^{2}}{\left(\left(x_{i}-x\right)^{2}+y^{2}+\left(z_{i}-z\right)^{2}\right)^{\frac{3}{2}}} .
$$

The last second derivative $\partial^{2} \Phi / \partial x_{i} \partial z_{i}$ is finally evaluated

$$
\begin{aligned}
& \frac{\partial^{2} \Phi}{\partial x_{i} \partial z_{i}}=\frac{\partial}{\partial x_{i}} \frac{\partial \Phi}{\partial z_{i}}=\frac{\partial}{\partial x_{i}}\left(-\frac{z_{i}-z}{\sqrt{x^{2}+y^{2}+z^{2}}}-\frac{k_{z_{i}}}{k}\right) \\
& \frac{\partial^{2} \Phi}{\partial x_{i} \partial z_{i}}=-\frac{\left(x-x_{i}\right)\left(z_{i}-z\right)}{\left(\left(x_{i}-x\right)^{2}+y^{2}+\left(z_{i}-z\right)^{2}\right)^{\frac{3}{2}}} .
\end{aligned}
$$

These three derivatives are finally evaluated at the stationary phase point

$$
\begin{aligned}
& \left.\frac{\partial^{2} \Phi}{\partial x_{i}^{2}}\right|_{x_{s}, z_{s}}=\frac{k^{2}-k_{x_{i}}^{2}}{y} \frac{\sqrt{k^{2}-k_{x_{i}}^{2}-k_{z_{i}}^{2}}}{k^{3}} \\
& \left.\frac{\partial^{2} \Phi}{\partial z_{i}^{2}}\right|_{x_{s}, z_{s}}=\frac{k^{2}-k_{z_{i}}^{2}}{y} \frac{\sqrt{k^{2}-k_{x_{i}}^{2}-k_{z_{i}}^{2}}}{k^{3}} .
\end{aligned}
$$

Finally

$$
\begin{aligned}
\left.\frac{\partial^{2} \Phi}{\partial x_{i} \partial z_{i}}\right|_{x_{s}, z_{s}} & =-\frac{\left(x-x_{s}\right)\left(z_{s}-z\right)}{\left(\left(x_{s}-x\right)^{2}+y^{2}+\left(z_{s}-z\right)^{2}\right)^{\frac{3}{2}}} \\
& =y^{-1} k^{-3} k_{x_{i}} k_{z_{i}}\left(k^{2}-k_{x_{i}}^{2}-k_{z_{i}}^{2}\right)^{\frac{1}{2}} .
\end{aligned}
$$

It is then required to evaluate $\left(\left.\left(\partial^{2} \Phi / \partial x_{i} \partial z_{i}\right)\right|_{x_{s}, z_{s}}\right)^{2}$

$$
\left(\left.\frac{\partial^{2} \Phi}{\partial x_{i} \partial z_{i}}\right|_{x_{s}, z_{s}}\right)^{2}=y^{-2} k^{-6} k_{x_{i}}^{2} k_{z_{i}}^{2}\left(k^{2}-k_{x_{i}}^{2}-k_{z_{i}}^{2}\right) .
$$

Finally, it is necessary to evaluate the expression of the amplitude term $R\left(x_{s}, z_{s}\right)$, as well as the expression of the phase term at the stationary point $\Phi\left(x_{s}, z_{s}\right)$

$$
\begin{aligned}
R\left(x_{s}, z_{s}\right) & =\sqrt{\left(x_{s}-x\right)^{2}+y^{2}+\left(z_{s}-z\right)^{2}} \\
& =\frac{k y}{\sqrt{k^{2}-k_{x_{i}}^{2}-k_{z_{i}}^{2}}} \\
\Phi\left(x_{s}, z_{s}\right) & =-\sqrt{\left(x_{s}-x\right)^{2}+y^{2}+\left(z_{s}-z\right)^{2}}-\frac{k_{x_{i}}}{k} x_{s}-\frac{k_{z_{i}}}{k} z_{s} \\
& =-\frac{k-\left(k_{x_{i}}^{2} / k\right)-\left(k_{z_{i}}^{2} / k\right)}{\sqrt{k^{2}-k_{x_{i}}^{2}-k_{z_{i}}^{2}}} y-\frac{k_{x_{i}}}{k} x-\frac{k_{z_{i}}}{k} z .
\end{aligned}
$$

The magnitude and phase term evaluated at the stationary point can then be extracted from the integral

$$
E_{i}=\frac{e^{j k \Phi\left(x_{s}, z_{s}\right)}}{R\left(x_{s}, z_{s}\right)} I_{g}
$$

where $I_{g}$ is a Gaussian integral evaluated around the stationary phase point [34], [35]

$$
\begin{aligned}
I_{g}=\int_{x_{i}} \int_{z_{i}} \exp (j k & \frac{1}{2}\left[\alpha\left(x_{i}-x_{s}\right)^{2}+\beta\left(z_{i}-z_{s}\right)^{2}\right. \\
& \left.\left.+2 \gamma\left(x_{i}-x_{s}\right)\left(z_{i}-z_{s}\right)\right]\right) \partial x_{i} \partial z_{i}
\end{aligned}
$$

with $\alpha=-\left.\frac{\partial^{2} \Phi}{\partial x_{i}^{2}}\right|_{x_{s}, z_{s}}, \beta=-\left.\frac{\partial^{2} \Phi}{\partial z_{i}^{2}}\right|_{x_{s}, z_{s}}$ and $\gamma=-\left.\frac{\partial^{2} \Phi}{\partial x_{i} \partial z_{i}}\right|_{x_{s}, z_{s}}$. Having $\alpha \beta>\gamma^{2}$ and $\alpha<0$, (64) then takes the following form [35]:

$$
\begin{aligned}
E_{i}= & -\frac{j 2 \pi}{k} R\left(x_{s}, z_{s}\right)^{-1} e^{j k \Phi\left(x_{s}, y_{s}\right)} \\
= & \left.-\left.\left.\frac{j 2 \pi}{k} \frac{\sqrt{k^{2}-k_{x_{i}}^{2}-k_{z_{i}}^{2}}}{k x_{i}^{2}}\right|_{x_{s}, z_{s}} \frac{\partial^{2} \Phi}{\partial z_{i}^{2}}\right|_{x_{s}, z_{s}}-\left(\left.\frac{\partial^{2} \Phi}{\partial x_{i} \partial z_{i}}\right|_{x_{s}, z_{s}}\right)^{2}\right)^{-\frac{1}{2}} \\
& \exp \left(-j\left(\frac{k^{2}-k_{x_{i}}^{2}-k_{z_{i}}^{2}}{\sqrt{k^{2}-k_{z_{i}}^{2}-k_{z_{i}}^{2}}} y+k_{x_{i}} x+k_{z_{i}} z\right)\right) \\
= & \frac{-j 2 \pi}{\sqrt{k^{2}-k_{x_{i}}^{2}-k_{z_{i}}^{2}}} \exp \left(-j \sqrt{k^{2}-k_{x_{i}}^{2}-k_{z_{i}}^{2}} y\right) \\
& \exp \left(-j k_{x_{i}} x\right) \exp \left(-j k_{z_{i}} z\right) .
\end{aligned}
$$

Finally, we recall the initial expression of the MIMO signal $S\left(k_{x_{t}}, k_{z_{t}}, k_{x_{r}}, k_{z_{r}}, k\right)$ expressed in the $k$-space, as well as its equivalent expression obtained by applying the stationary phase method

$$
\begin{aligned}
S= & \int_{r} \frac{\sigma(r)}{16 \pi^{2}}\left(\int_{A_{t}} \frac{e^{-j k R_{t}}}{R_{t}} e^{j k_{x_{t}} x_{t}} e^{j k_{z t} z t} d A_{t}\right) \\
& \left(\int_{A_{r}} \frac{e^{-j k R_{r}}}{R_{r}} e^{j k_{x_{r}} x_{r}} e^{j k_{z r} z_{r}} d A_{r}\right) d^{3} r \\
= & \frac{4 \pi^{2}}{16 \pi^{2} k_{y_{t}} k_{y_{r}}} \int_{r} \sigma(r) e^{-j k_{y_{t}} y} e^{-j k_{x_{t}} x} e^{-j k_{z_{t}} z} \\
= & \frac{1}{4 k_{y_{t}} k_{y_{r}}} \int_{r} \sigma(r) e^{-j k_{y_{r}} y} e^{-j k_{x_{r}} x} e^{-j k_{z_{r}} z} d^{3} r
\end{aligned}
$$

where the association of transverse components corresponding to the plane waves emitted and received gives rise to new projections of composite wave vectors interrogating the target space, matching the expressions given in [13]

$$
\begin{aligned}
k_{y_{t}} & =\sqrt{k^{2}-k_{x_{t}}^{2}-k_{z_{t}}^{2}} \\
k_{y_{r}} & =\sqrt{k^{2}-k_{x_{r}}^{2}-k_{z_{r}}^{2}} \\
k_{x} & =k_{x_{t}}+k_{x_{r}} \\
k_{y} & =k_{y_{t}}+k_{y_{r}} \\
k_{z} & =k_{z_{t}}+k_{z_{r}} .
\end{aligned}
$$

\section{ACKNOWLEDGMENT}

The authors would like to thank the anonymous reviewers whose relevant comments helped to improve the content of this paper. T. Fromenteze would like to thank the online translator DeepL for facilitating the writing of several sections of this paper. 


\section{REFERENCES}

[1] B. D. Steinberg and H. M. Subbaram, Microwave Imaging Techniques. New York, NY, USA: Wiley, 1991

[2] D. W. Bliss and K. W. Forsythe, "Multiple-input multiple-output (MIMO) radar and imaging: Degrees of freedom and resolution," in Proc. IEEE Conf. Rec. 37th Asilomar Conf. Signals, Syst. Comput., vol. 1, Nov. 2003, pp. 54-59.

[3] Y. Yu, A. P. Petropulu, and H. V. Poor, "Measurement matrix design for compressive sensing-based MIMO radar," IEEE Trans. Signal Process., vol. 59, no. 11 , pp. 5338-5352, Nov. 2011.

[4] M. Rossi, A. M. Haimovich, and Y. C. Eldar, "Spatial compressive sensing for MIMO radar," IEEE Trans. Signal Process., vol. 62, no. 2, pp. 419-430, Jan. 2014

[5] Y. Álvarez, Y. Rodriguez-Vaqueiro, B. Gonzalez-Valdes, F. Las-Heras, and A. García-Pino, "Fourier-based imaging for subsampled multistatic arrays," IEEE Trans. Antennas Propag., vol. 64, no. 6, pp. 2557-2562, Jun. 2016.

[6] D. Carsenat and C. Decroze, "UWB antennas beamforming using passive time-reversal device," IEEE Antennas Wireless Propag. Lett., vol. 11, pp. 779-782, 2012

[7] J. Hunt et al., "Metamaterial apertures for computational imaging," Science, vol. 339, no. 6117, pp. 310-313, Jan. 2013.

[8] Y. Álvarez et al., "Submillimeter-wave frequency scanning system for imaging applications," IEEE Trans. Antennas Propag., vol. 61, no. 11, pp. 5689-5696, Nov. 2013.

[9] T. Fromenteze, C. Decroze, and D. Carsenat, "Waveform coding for passive multiplexing: Application to microwave imaging," IEEE Trans. Antennas Propag., vol. 63, no. 2, pp. 593-600, Feb. 2015.

[10] T. Fromenteze et al., "Computational imaging using a mode-mixing cavity at microwave frequencies," Appl. Phys. Lett., vol. 106, no. 19, 2015, Art. no. 194104.

[11] J. Fortuny-Guasch and J. M. Lopez-Sanchez, "Extension of the 3-D range migration algorithm to cylindrical and spherical scanning geometries," IEEE Trans. Antennas Propag., vol. 49, no. 10, pp. 1434-1444, Oct. 2001

[12] J. M. Lopez-Sanchez and J. Fortuny-Guasch, "3-D radar imaging using range migration techniques," IEEE Trans. Antennas Propag., vol. 48, no. 5, pp. 728-737, May 2000.

[13] X. Zhuge and A. G. Yarovoy, "Three-dimensional near-field MIMO array imaging using range migration techniques," IEEE Trans. Image Process., vol. 21, no. 6, pp. 3026-3033, Jun. 2012.

[14] T. Fromenteze, E. L. Kpré, D. Carsenat, C. Decroze, and T. Sakamoto, "Single-shot compressive multiple-inputs multiple-outputs radar imaging using a two-port passive device," IEEE Access, vol. 4, pp. 1050-1060, 2016.

[15] L. Pulido-Mancera et al., "Application of range migration algorithms to imaging with a dynamic metasurface antenna," J. Opt. Soc. Amer. B, Opt. Phys., vol. 33, no. 10, pp. 2082-2092, 2016.

[16] T. Fromenteze, X. Liu, M. Boyarsky, J. Gollub, and D. R. Smith, "Phaseless computational imaging with a radiating metasurface," Opt. Express, vol. 24, no. 15, p. 16760-16776, 2016.

[17] T. Fromenteze, O. Yurduseven, M. Boyarsky, J. Gollub, D. L. Marks, and D. R. Smith, "Computational polarimetric microwave imaging," Opt. Express, vol. 25, no. 22, pp. 27488-27505, 2017.

[18] O. Yurduseven, T. Fromenteze, D. L. Marks, J. N. Gollub, and D. R. Smith, "Frequency-diverse computational microwave phaseless imaging," IEEE Antennas Wireless Propag. Lett., vol. 16, pp. 2808-2811, 2017.

[19] S. S. Ahmed, Electronic Microwave Imaging With Planar Multistatic Arrays. Berlin, Germany: Logos Verlag Berlin GmbH, 2014.

[20] S. S. Ahmed, "Advanced fully-electronic personnel security screening technology," in Proc. IEEE 9th Eur. Conf. Antennas Propag. (EuCAP), Apr. 2015, pp. 1-4.

[21] Q. Guo, J. Wang, T. Chang, and H.-L. Cui, "Dimension-factorized range migration algorithm for regularly distributed array imaging," Sensors, vol. 17, no. 11, p. 2549, 2017.

[22] E. L. Kpré, T. Fromenteze, and C. Decroze, "MIMO radar transmit array fed by a $1 \times \mathrm{M}$ passive chaotic cavity," in Proc. IEEE 10th Eur. Conf. Antennas Propag. (EuCAP), Apr. 2016, pp. 1-5.

[23] D. Gabor, "A new microscopic principle," Nature, vol. 161, no. 4098 , pp. $777-778,1948$

[24] M. Takeda, H. Ina, and S. Kobayashi, "Fourier-transform method of fringe-pattern analysis for computer-based topography and interferometry," J. Opt. Soc. Amer. A, Opt. Image Sci., vol. 72, no. 1, pp. 156-160, 1982.
[25] D. M. Meadows, W. O. Johnson, and J. B. Allen, "Generation of surface contours by Moiré patterns," Appl. Opt., vol. 9, no. 4, pp. 942-947, 1970.

[26] G. Lipworth et al., "Comprehensive simulation platform for a metamaterial imaging system," Appl. Opt., vol. 54, no. 31, pp. 9343-9353, 2015.

[27] J. N. Gollub et al., "Large metasurface aperture for millimeter wave computational imaging at the human-scale," Sci. Rep., vol. 7, Feb. 2017, Art. no. 42650

[28] R. F. Harrington, Time-Harmonic Electromagnetic Fields. New York, NY, USA: McGraw-Hill, 1961

[29] D. L. McMakin, D. M. Sheen, and T. E. Hall, "Holographic arrays for multi-path imaging artifact reduction," U.S. Patent 7295146 , Nov. 13, 2007.

[30] B. Gonzalez-Valdes, Y. Alvarez-Lopez, J. A. Martinez-Lorenzo, F. L. H. Andres, and C. M. Rappaport, "SAR processing for profile reconstruction and characterization of dielectric objects on the human body surface," Prog. Electromagn. Res., vol. 138, pp. 269-282, Mar. 2013.

[31] M. Ibrahim and O. Khan, "Performance analysis of fast Fourier transform on field programmable gate arrays and graphic cards," in Proc. IEEE Int. Conf. Comput., Electron. Elect. Eng. (ICE Cube), Apr. 2016, pp. $158-162$.

[32] Y. Alvarez et al., "Fourier-based imaging for multistatic radar systems," IEEE Trans. Microw. Theory Techn., vol. 62, no. 8, pp. 1798-1810, Aug. 2014.

[33] J. M. L. Sánchez and M. B. Escudero, Análisis y Estimación de Parámetros Físicos de Vegetación Mediante Polarimetría Radar. Valencia, Spain: Univ. Valencia, 1999, p. 140.

[34] N. G. Van Kampen, "The method of stationary phase and the method of Fresnel zones," Physica, vol. 24, nos. 1-5, pp. 437-444, 1958.

[35] M. Born and E. Wolf, Principles of Optics: Electromagnetic Theory of Propagation, Interference and Diffraction of Light. Amsterdam, The Netherlands: Elsevier, 2013.

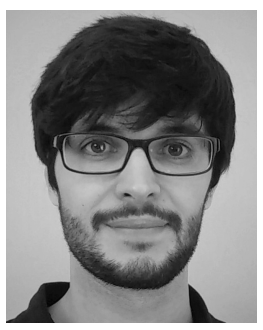

Thomas Fromenteze received the Ph.D. degree from the University of Limoges, Limoges, France, in 2015

From 2015 to 2016, he was a Post-Doctoral Researcher with Duke University, Durham, NC, USA. He is currently a Maître de Conférences (Assistant Professor) with the Xlim Research Institute, University of Limoges, Limoges, France. He is also an Adjunct Assistant Professor with the Center for Metamaterials and Integrated Plasmonics, Duke University. His research interests include ultrawideband microwave and millimeter-wave imaging, wave propagation in complex media, computational/compressive imaging, and the various associated inverse problems.

Dr. Fromenteze received the 11th EuRAD Young Engineer Prize during the European Microwave Week 2015.



Okan Yurduseven (S'09-M'11-SM'16) received the B.Sc. and M.Sc. degrees in electrical engineering from Yildiz Technical University, Istanbul, Turkey, in 2009 and 2011, respectively, and the Ph.D. degree in electrical engineering from Northumbria University, Newcastle upon Tyne, U.K., in 2014.

From 2018 to 2019, he was a NASA Post-Doctoral Fellow with the Jet Propulsion Laboratory, California Institute of Technology, Pasadena, CA, USA. From 2014 to 2018, he was a Post-Doctoral Research Associate with the Department of Electrical and Computer Engineering, Duke University, working in collaboration with the U.S. Department of Homeland Security. He is currently a Senior Lecturer (Associate Professor) with the Centre for Wireless Innovation (CWI), School of Electronics, Electrical Engineering and Computer Science, Institute of Electronics, Communications and Information, Technology, Queen's University Belfast, Belfast, U.K. He is also an Adjunct Assistant Professor with Duke University, Durham, NC, USA. His research interests include microwave and millimeter-wave imaging, multiple-input-multiple-output (MIMO) radar, wireless power transfer, antennas and propagation, antenna measurement techniques, and metamaterials. He has authored more than 100 peer-reviewed technical journal and conference articles. 
Dr. Yurduseven is a member of the European Association on Antennas and Propagation (EurAAP). He has organized and chaired numerous sessions in international symposiums and conferences, including the IEEE International Symposium on Antennas and Propagation (AP-S) and the European Conference on Antennas and Propagation (EuCAP). He was a recipient of the Academic Excellence Award from the Association of British Turkish Academics (ABTA) in London, in 2013. He received the Best Paper Award from the Mediterranean Microwave Symposium in 2012, the Travel Award from the Institution of Engineering and Technology (IET), a NASA Postdoctoral Program Fellowship administrated by the Universities Space Research Association under contract with NASA in 2017, and the Outstanding Postdoctoral Award from Duke University and the Duke Postdoctoral Professional Development Award in 2017.

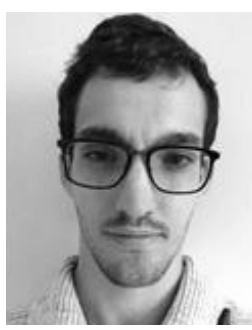

Fabien Berland received the M.S degree in microwave engineering from the University of Limoges, Limoges, France, in 2018, where he is currently pursuing the Ph.D. degree with the Xlim Research Institute.

His research interests include radar imaging and microwave photonics systems.

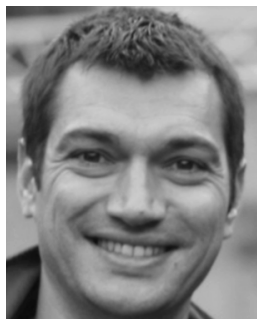

Cyril Decroze received the $\mathrm{Ph} . \mathrm{D}$. degree in telecommunications engineering from the University of Limoges, Limoges, France, in 2002, and HDR degree from the XLIM Research Institute in 2013.

Since 2015, he has been in charge of the Antennas and Signal Team, XLIM. He is currently an Associate Professor with the XLIM Research Institute. His activities are supported by a strong experimental part, including test-benches development, allowing active characterization of antennas processing algorithms. He participated in four national research funding projects (including one as a Coordinator), three European projects (including two as a Scientific Leader), and several industrial contracts. $\mathrm{He}$ has co-supervised $15 \mathrm{Ph} . \mathrm{D}$. thesis (five ongoing). He has co-authored 32 publications in international journals, 46 international communications, and four patents. His research interests include multiple antennas' transmission systems and associated processing for communications and radar, and channel sounding and channel emulation.



Alexander G. Yarovoy (F'15) received the Diploma degree (Hons.) in radiophysics and electronics and the Candidate Phys. and Math. Sci. and Doctor Phys. and Math. Sci. degrees in radiophysics from Kharkov State University, Kharkov, Ukraine, in 1984,1987 , and 1994, respectively.

In 1987, he joined the Department of Radiophysics, Kharkov State University, as a Researcher, where he became a Professor in 1997. From 1994 to 1996, he was a Visiting Researcher with the Technical University of Ilmenau, Ilmenau, Germany. Since 1999, he has been with the Delft University of Technology, Delft, The Netherlands, where he has been the Chair of the Microwave Sensing, Systems and Signals since 2009. His research interests include high-resolution radar, microwave imaging, and applied electromagnetics (in particular, ultrawideband antennas). He has authored or co-authored more than 450 scientific or technical papers and fourteen book chapters. He holds four patents.

Dr. Yarovoy was a recipient of the European Microwave Week Radar Award for the paper that best advances the state of the art in radar technology in 2001 (together with L. P. Ligthart and P. van Genderen) and in 2012 (together with T. Savelyev). In 2010, together with D. Caratelli, he received the Best Paper Award of the Applied Computational Electromagnetic Society (ACES). He served as the Chair and the TPC Chair of the 5th European Radar Conference (EuRAD 2008), Amsterdam, The Netherlands, and the Secretary of the 1st European Radar Conference (EuRAD 2004), Amsterdam. He served as the Co-Chair and the TPC Chair of the Xth International Conference on GPR (GPR 2004), Delft. From 2008 to 2017, he was the Director of the European Microwave Association (EuMA). He served as a Guest Editor of five special issues for the IEEE TRANSACTIONS and other journals. Since 2011, he has been an Associate Editor of the International Journal of Microwave and Wireless Technologies.

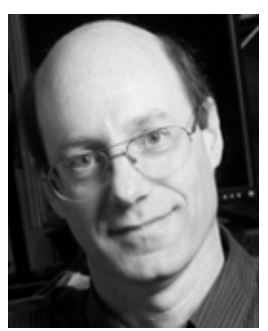

David R. Smith (M'03) received the B.S. and Ph.D. degrees in physics from the University of California at San Diego, San Diego, CA, USA, in 1988 and 1994, respectively.

$\mathrm{He}$ is currently the Department Chair and a James B. Duke Professor of electrical and computer engineering with Duke University and the Director of the Center for Metamaterials and Integrated Plasmonics, Duke University, Durham, NC, USA. $\mathrm{He}$ is also an Adjunct Professor with the Physics Department, University of California at San Diego, an Affiliate Faculty Member with the Electrical and Computer Engineering Department, University of Washington, Seattle, WA, USA, and a Visiting Professor of physics with Imperial College, London, U.K. His research interests include the theory, simulation, and characterization of unique electromagnetic structures, including photonic crystals and metamaterials, and applications of such materials. 